\title{
Correspondence
}

\section{Channel Aware Decision Fusion in Wireless Sensor Networks}

\author{
Biao Chen, Ruixiang Jiang, Teerasit Kasetkasem, and \\ Pramod K. Varshney
}

\begin{abstract}
Information fusion by utilizing multiple distributed sensors is studied in this work. Extending the classical parallel fusion structure by incorporating the fading channel layer that is omnipresent in wireless sensor networks, we derive the likelihood ratio based fusion rule given fixed local decision devices. This optimum fusion rule, however, requires perfect knowledge of the local decision performance indices as well as the fading channel. To address this issue, two alternative fusion schemes, namely, the maximum ratio combining statistic and a two-stage approach using the Chair-Varshney fusion rule, are proposed that alleviate these requirements and are shown to be the low and high signal-to-noise ratio (SNR) equivalents of the likelihood-based fusion rule. To further robustify the fusion rule and motivated by the maximum ratio combining statistics, we also propose a statistic analogous to an equal gain combiner that requires minimum $a$ priori information. Performance evaluation is performed both analytically and through simulation.
\end{abstract}

Index Terms-Decision fusion, diversity combining, fading channel, wireless sensor networks.

\section{INTRODUCTION}

Wireless sensor networks (WSNs) have generated intensive interest from the research community in the past few years. Typical applications include battlefield surveillance, environment, and structure monitoring, among others. In battlefield surveillance applications, microsensors are to be deployed in large numbers to form an intensive information network [1], [2]. Communication capabilities are integrated into each sensor node to allow transmission of the locally processed information to a central node, where information from various sensors is processed collectively to form a final situation assessment.

Much of current research effort on WSN is focused on the development of energy efficient routing protocols, distributed data compression and transmission schemes, and collaborative signal processing algorithms, as documented in [2] and references therein. The fact that locally processed information is transmitted through a fading channel has not attracted much attention. While channel fades may be treated purely as a communication issue and thus be dealt with exclusively through transceiver design, its effect on the signal processing task at the fusion center has not been carefully considered. Our attempt in this correspondence is to incorporate the communication aspects into the data processing stage and to design signal processing algorithms, in particular, a decision fusion algorithm, that can intelligently cope with channel fades at the decision-making stage.

Manuscript received August 18, 2003; revised December 12, 2003. This work was presented in part at the 36th Asilomar Conference on Signals, Systems, and Computing, Pacific Grove, CA, 2002. The associate editor coordinating the review of this manuscript and approving it for publication was Dr. Zhi-Quan (Tom) Luo.

B. Chen, R. Jiang, and P. K. Varshney are with the Department of Electrical Engineering and Computer Science, Syracuse University, Syracuse, NY 13244 USA (e-mail: bichen@ecs.syr.edu; rjiang@ecs.syr.edu; varshney@ecs.syr.edu).

T. Kasetkasem is with Electrical Engineering Department, Kasetsart University, Chatuchak, Bangkok, 10900 Thailand (e-mail: fengtsk@ku.ac.th).

Digital Object Identifier 10.1109/TSP.2004.837404
The problem of distributed detection, and in particular, decision fusion, has been studied extensively in the past decades. In the absence of fading channel consideration, optimum fusion rules have been obtained for both binary and multibit (soft) local sensor output under the conditional independence assumption [3], [4]. Fusion rules with statistically correlated observations have also been investigated though the results are considerably more involved [5], [6] and are mostly limited to binary decisions. Decision fusion with uncertainty has also been investigated and a Bayesian sampling approach has been proposed to address this issue [7]. Decision fusion has also been considered under some communication constraint [8]-[12], where the communication constraint is often in the form of total number of bits allowed to be transmitted from local sensors to the reciever without regard to the possible channel induced transmission errors. Decision fusion with nonideal channels has been investigated by Thomopoulos and Zhang [13]. where binary symmetric channels are used to model the transmission of local decisions to the fusion center. Person by person optimization is used in deterimining the likelihood ratio thresholds for both the local sensors and the fusion center. A similar problem is investigated in Duman and Salehi [14] where a multiple access channel (MAC) is used to model the collective transmission from multiple sensors to a fusion center and a more general multilevel quantization at local sensors is treated.

In this correspondence, we explore decision fusion algorithms that take into account channel fading effects. While it can be argued that at low-rate transmission, the physical layer can achieve arbitrary reliability, it is always desirable to reduce the communication power in energy constrained systems. This motivates designs that can achieve good fusion performance with minimum amount of power consumption. In other words, there is always an incentive in pushing the energy consumption to its minimum possible value for expanded sensor network lifetime. It will become clear later in this correspondence that channel-aware fusion strategies are much more energy efficient in this sense than the conventional two-stage approach, where communication and fusion are treated as independent processes. In addition, for sensor networks operating in a hostile environment, the transmitted signal should be kept at minimum power for low probability of intercept/detection (LPI/LPD).

The organization of this correspondence is as follows. In the next section we formulate the parallel fusion problem with a fading channel layer and derive the optimal likelihood ratio (LR)-based fusion rule with binary local decisions. The three alternative fusion rules are also presented: a two-stage approach using the Chair-Varshney fusion rule, a maximum ratio combining (MRC) fusion statistic, and an equal gain combiner (EGC) fusion statistic. We show that the first two schemes are respectively high and low signal-to-noise ratio (SNR) approximations of the LR-based fusion rule. Performance evaluation, including numerical examples, is contained in Section III. Assuming a Rayleigh flat fading channel model, the EGC statistic seems to provide the most robust fusion alternative among the three suboptimum fusion statistics while requiring minimum a priori information. We conclude in Section IV.

\section{DECISION Fusion Under FAding ChANNEL ASSUMPTION}

\section{A. Problem Formulation}

Fig. 1 depicts a typical parallel fusion structure where a number of sensors observe data generated according to either $\mathbf{H}_{0}$ (target-absent) or $\mathbf{H}_{1}$ (target-present), which are the two hypotheses under test. Each 


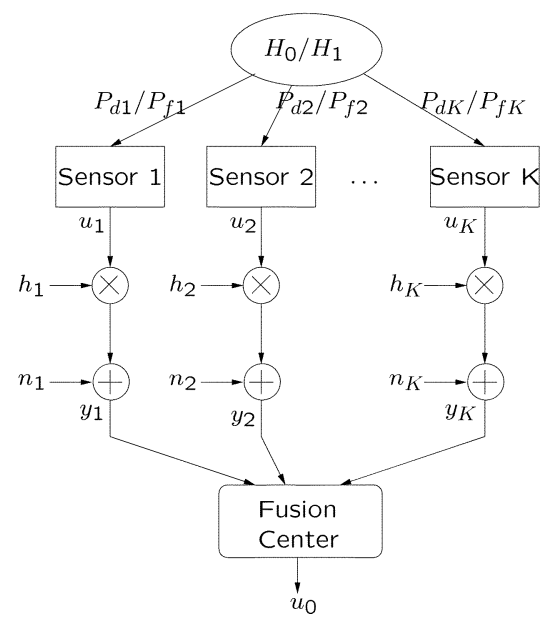

Fig. 1. Parallel fusion model in the presence of fading and noisy channel between local sensors and the fusion center.

sensor processes its observations and makes a preliminary decision about the hypothesis before sending it to a fusion center. The incorporation of the fading channel layer suggests that the local decisions may be subject to transmission errors. Incorrectly received local decisions will lead to performance loss. This loss can be minimized by properly considering and including the effect of channel impairments in the derivation of the decision fusion rule.

Assume that the $k$ th local sensor makes a binary decision $u_{k} \in$ $\{+1,-1\}$, with false alarm and detection probabilities $P_{f k}$ and $P_{d k}$, respectively. That is, $P\left[u_{k}=1 \mid \mathbf{H}_{0}\right]=P_{f k}$ and $P\left[u_{k}=1 \mid \mathbf{H}_{1}\right]=$ $P_{d k}$. From Fig. 1, each local decision $u_{k}$ is transmitted through a fading channel and the output of the channel (or input to the fusion center) for the $k$ th sensor is

$$
y_{k}=h_{k} u_{k}+n_{k}
$$

where $h_{k}$ is a real valued fading envelope with $h_{k}>0$, and $n_{k}$ is zero mean Gaussian noise with variance $\sigma^{2}$. The above model assumes phase coherent reception, which can be either accomplished through training-based channel estimation for stationary channels or at a small cost of SNR degradation, by employing differential encoding for a fast fading channel. Our goal now is to derive a fusion rule based on $y_{k}$, for $k=1, \ldots, K$, that can determine which hypothesis is true with the best achievable performance.

\section{B. Optimal LR-Based Fusion Rule}

Assuming complete knowledge regarding the fading channel and the local sensor performance indices, i.e., the $P_{f k}$ and $P_{d k}$ values, the LR at the fusion center, given the conditional independence assumption of local observations, can be easily derived as

$$
\begin{aligned}
\Lambda(\mathbf{y}) & =\frac{f\left(\mathbf{y} \mid H_{1}\right)}{f\left(\mathbf{y} \mid H_{0}\right)} \\
& =\prod_{k=1}^{K} \frac{P_{d k} e^{-\frac{\left(y_{k}-h_{k}\right)^{2}}{2 \sigma^{2}}}+\left(1-P_{d k}\right) e^{-\frac{\left(y_{k}+h_{k}\right)^{2}}{2 \sigma^{2}}}}{P_{f k} e^{-\frac{\left(y_{k}-h_{k}\right)^{2}}{2 \sigma^{2}}}+\left(1-P_{f k}\right) e^{-\frac{\left(y_{k}+h_{k}\right)^{2}}{2 \sigma^{2}}}}
\end{aligned}
$$

where $\mathbf{y}=\left[y_{1}, \ldots, y_{K}\right]^{T}$ is a vector containing observations received from all $K$ sensors. While the form of the LR-based fusion rule is straightforward to implement, it does need both the local sensor performance indices and complete channel knowledge. In the next section, we investigate a number of suboptimum fusion rules that relieve the above requirements to a certain extent.

\section{Suboptimum Fusion Rules}

The first two alternatives are proposed as high and low SNR approximations to the LR fusion rule that partially relieve some of the requirements associated with the LR-based fusion rule. This is established by showing that the log likelihood nonlinearity converges to the two respective forms under the high and low SNR assumptions. The fact that these alternatives have familiar forms in the context of distributed detection and diversity combining provides some important insights. Motivated by the form of the low SNR alternative, we propose another statistic that requires minimum a priori information and, as it turns out, enjoys the most robust performance among the three suboptimal approaches for a wide range of channel SNR values.

1) Chair-Varshney Fusion Rule-Based Two-Stage Approximation: The fusion rule specified in (2) jointly considers the effects of the fading channel and the local sensor outputs to achieve optimal performance. A direct alternative is to separate this into a two stage process-first, $y_{k}$ is used to infer about $u_{k}$, and then, the optimum fusion rule based on $u_{k}$ (assuming that the estimates are reliable), as derived in [3], can be applied. Given (1) and with $u_{k} \in\{1,-1\}$, the maximum likelihood (ML) estimate for $u_{k}$ is simply

$$
\hat{u}_{k}=\operatorname{sign}\left(y_{k}\right) \text {. }
$$

Applying the fusion rule derived in [3], herein termed the Chair-Varshney fusion rule, we obtain the following statistic:

$$
\Lambda_{1}=\sum_{\operatorname{sign}\left(y_{k}\right)=1} \log \frac{P_{d k}}{P_{f k}}+\sum_{\operatorname{sign}\left(y_{k}\right)=-1} \log \frac{1-P_{d k}}{1-P_{f k}} .
$$

For large-channel SNR, the ML estimate of $u_{k}$ is most likely to be correct and this statistic should be a good approximation for $\Lambda$. Indeed, we show below that, as $\sigma^{2} \rightarrow 0$, the logarithm of $\Lambda$ converges to $\Lambda$, i.e.,

$$
\lim _{\sigma^{2} \rightarrow 0} \log \Lambda=\Lambda_{1}
$$

To show this, we define $\mathcal{S}_{0}=\left\{k: y_{k}<0\right\}$ and $\mathcal{S}_{1}=\left\{k: y_{k}>0\right\}$, and we rewrite the LR-based fusion statistic as

$$
\begin{aligned}
\Lambda=\prod_{k \in \mathcal{S}_{0}} \frac{P_{d k}+\left(1-P_{d k}\right) e^{-\frac{2 y_{k} h_{k}}{\sigma^{2}}}}{P_{f k}+\left(1-P_{f k}\right) e^{-\frac{2 y_{k} h_{k}}{\sigma^{2}}}} \\
\quad \times \prod_{k \in \mathcal{S}_{1}} \frac{P_{d k} e^{\frac{2 y_{k} h_{k}}{\sigma^{2}}}+\left(1-P_{d k}\right)}{P_{f k} e^{\frac{2 y_{k} h_{k}}{\sigma^{2}}}+\left(1-P_{f k}\right)} .
\end{aligned}
$$

Given that $\sigma^{2} \rightarrow 0$ (high SNR assumption), we have, for $k \in$ $\mathcal{S}_{0}, e^{-\left(2 y_{k} h_{k}\right) /\left(\sigma^{2}\right)} \gg 1$, whereas for $k \in \mathcal{S}_{1}, e^{\left(2 y_{k} h_{k}\right) /\left(\sigma^{2}\right)} \gg 1$. Hence, the assumption $\sigma^{2} \rightarrow 0$ can be used to obtain

$$
\lim _{\sigma^{2} \rightarrow 0} \Lambda=\prod_{k \in \mathcal{S}_{0}} \frac{1-P_{d k}}{1-P_{f k}} \prod_{k \in \mathcal{S}_{1}} \frac{P_{d k}}{P_{f k}} .
$$

Taking logarithm of both sides, we get

$$
\lim _{\sigma^{2} \rightarrow 0} \log \Lambda=\sum_{k \in \mathcal{S}_{0}} \log \left[\frac{1-P_{d k}}{1-P_{f k}}\right]+\sum_{k \in \mathcal{S}_{1}} \log \left[\frac{P_{d k}}{P_{f k}}\right]=\Lambda_{1}
$$

which is precisely the decision statistic of the optimum fusion rule derived in [3].

Notice that $\Lambda_{1}$ does not require any knowledge regarding the channel statistics but does require $P_{d k}$ and $P_{f k}$ for all $k$. Notice also that at low SNR, because of the increased likelihood of making an error in inferring $u_{k}$ from $y_{k}$, performance degradation is expected using this two-stage approach. 
2) Maximum Ratio Combining Fusion Statistic: We start by simplifying $\Lambda$ in a similar fashion:

$$
\Lambda=\prod_{k=1}^{K} \frac{P_{d k}+\left(1-P_{d k}\right) e^{-\frac{2 y_{k} h_{k}}{\sigma^{2}}}}{P_{f k}+\left(1-P_{f k}\right) e^{-\frac{2 y_{k} h_{k}}{\sigma^{2}}}} .
$$

For low SNR, i.e., $\sigma^{2} \rightarrow \infty$, we have $e^{-\left(2 y_{k} h_{k}\right) /\left(\sigma^{2}\right)} \rightarrow 1$, which can be approximated by the first-order Taylor series expansion, i.e., $e^{-\left(2 y_{k} h_{k}\right) /\left(\sigma^{2}\right)} \approx 1-\left(2 y_{k} h_{k}\right) /\left(\sigma^{2}\right)$. Therefore, we have

$$
\begin{aligned}
\lim _{\sigma^{2} \rightarrow \infty} \Lambda & =\lim _{\sigma^{2} \rightarrow \infty} \prod_{k=1}^{K} \frac{P_{d k}+\left(1-P_{d k}\right)\left(1-\frac{2 y_{k} h_{k}}{\sigma^{2}}\right)}{P_{f k}+\left(1-P_{f k}\right)\left(1-\frac{2 y_{k} h_{k}}{\sigma^{2}}\right)} \\
& =\lim _{\sigma^{2} \rightarrow \infty} \prod_{k=1}^{K} \frac{1-\left(1-P_{d k}\right) \frac{2 y_{k} h_{k}}{\sigma^{2}}}{1-\left(1-P_{f k}\right) \frac{2 y_{k} h_{k}}{\sigma^{2}}} .
\end{aligned}
$$

Taking logarithm of both sides, we get

$$
\begin{aligned}
\lim _{\sigma^{2} \rightarrow \infty} \log \Lambda=\lim _{\sigma^{2} \rightarrow \infty}\left\{\sum_{k=1}^{K} \log \right. & {\left[1-\left(1-P_{d k}\right) \frac{2 y_{k} h_{k}}{\sigma^{2}}\right] } \\
& \left.-\sum_{k=1}^{K} \log \left[1-\left(1-P_{f k}\right) \frac{2 y_{k} h_{k}}{\sigma^{2}}\right]\right\} .
\end{aligned}
$$

Using the fact that, for $x \rightarrow 0$

$$
\log (1+x)=x+o(x)
$$

where $o(x)$ denotes a term with $\lim _{x \rightarrow 0}(o(x)) /(x)=0$, we have the following equivalence in the limit:

$$
\begin{aligned}
\lim _{\sigma^{2} \rightarrow \infty} \log \Lambda= & \lim _{\sigma^{2} \rightarrow \infty}\left[-\sum_{k=1}^{K}\left(1-P_{d k}\right) \frac{2 y_{k} h_{k}}{\sigma^{2}}\right. \\
& \left.+\sum_{k=1}^{K}\left(1-P_{f k}\right) \frac{2 y_{k} h_{k}}{\sigma^{2}}\right] \\
= & \lim _{\sigma^{2} \rightarrow \infty} \sum_{k=1}^{K}\left(P_{d k}-P_{f k}\right) \frac{2 y_{k} h_{k}}{\sigma^{2}} .
\end{aligned}
$$

Given that $\sigma^{2}$ is a (possibly unknown) constant and, hence, can be neglected, the above limiting statistic reduces to

$$
\hat{\Lambda}_{2}=\sum_{k=1}^{K}\left(P_{d k}-P_{f k}\right) h_{k} y_{k} .
$$

Further, if the local sensors are identical, i.e., $P_{d k}$ and $P_{f k}$ are the same for all $k$ 's, then $\Lambda$ reduces to a form analogous to a maximum ratio combiner [15]:

$$
\Lambda_{2}=\frac{1}{K} \sum_{k=1}^{K} h_{k} y_{k}
$$

The factor $1 / K$ in $\Lambda_{2}$ does not affect the detection performance but is introduced for the convenience of performance analysis. In essence, $\Lambda_{2}$ is the first-order approximation of the log likelihood ratio based fusion rule and is asymptotically accurate as $\sigma^{2} \rightarrow \infty$. Notice that $\Lambda_{2}$ does not require the knowledge of $P_{d k}$ and $P_{f k}$, provided $P_{d k}-P_{f k}>0$. Knowledge of the channel gain is, however, required.

3) Equal Gain Combining Fusion Statistic: Motivated by the fact that $\Lambda_{2}$ resembles a MRC statistic for diversity combining, we propose a third alternative in the simple form of an equal gain combiner (EGC) that requires minimum amount of information:

$$
\Lambda_{3}=\frac{1}{K} \sum_{k=1}^{K} y_{k}
$$

While this heuristic and the simple fusion rule in the form of an EGC statistic does relieve most of the requirements compared with the optimal LR-based fusion rule, its usefulness largely depends on its performance compared with the optimum fusion rule as well as the two alternatives proposed above. In the context of diversity combining for wireless communications, EGC is considered a suboptimal approach to MRC due to the fact that the latter maximizes the output SNR. Interestingly, we show that in the context of decision fusion for WSN, this simple alternative outperforms both $\Lambda_{1}$ and $\Lambda_{2}$ for a wide range of SNR in terms of its detection performance.

\section{Performance Evaluation}

In this section, we evaluate the detection performance of various fusion rules. Under the Neyman-Pearson criterion, it is clear that the LR-based fusion rule provides the best detection performance. The question is, therefore, how much performance degradation the other three simple alternatives suffer and, among these three, which one provides the most robust detection performance. We emphasize here that among the three suboptimal approaches, the EGC and MRC are perhaps more desirable because of their performance advantage compared with the two-stage approach for low to medium SNR values. Most wireless sensor networks are energy limited-once the sensors are deployed, the lifetime may depend solely on the on-board battery supply. Given that RF communication is the most energy consuming function of a sensor node, it is, therefore, imperative to use as little power as possible for data transmission, which usually results in modest SNR values at the fusion center receiver.

Throughout this section, we assume a Rayleigh fading model for both analysis and simulation. Other fading types, such as Ricean fading, can be used instead, although the analysis is more involved.

\section{A. Performance Analysis of MRC and EGC Fusion Rules}

While it is well known that MRC is optimal in that it maximizes the output SNR, it relies on an assumption that is taken for granted in wireless communications, that is, the sources for multiple independently faded channels are identical to each other. Under this condition, MRC achieves maximum output SNR as it involves full coherent combining. In the context of sensor networks, this is not necessarily the case; the local sensors are prone to decision errors due to the nature of the problem. Clearly, without identical input to the multiple fading channels, there is no guarantee that MRC is still preferable compared with other alternatives such as EGC.

The model specified in Section II-A, together with the simple forms of MRC and EGC, allows the development of numerical procedures for the evaluation of the fusion network performance in terms of the probabilities of detection and false alarm. We call them system level detection probability and false alarm rate, which is denoted by $P_{d 0}$ and $P_{f 0}$, to distinguish them from sensor level $P_{d k}$ and $P_{f k}$. These procedures usually involve multiple integration and do not lead to any insight regarding the performance discrepancy between different fusion rules. If we assume that the sensors are identical to each other (thus, $P_{f k}=P_{f}$ and $P_{d k}=P_{d}$ for all $k$ ), then both MRC and EGC fusion statistics are sums of independent and identically distributed random variables. This allows direct application of the CLT and the limiting distributions corresponding to the underlying hypotheses are Gaussian with respective mean and variance. Detection and false alarm probabilities hence can be evaluated fairly easily [16]. To do so, we need the first two moments of the test statistics under both hypotheses and they are summarized in Table I for Rayleigh fading channel. Derivation of these statistics is straightforward hence is skipped for brevity.

Fig. 2 gives the receiver operating characteristic (ROC) curves obtained both by Monte Carlo simulation and analytical approximation 
TABLE I

Mean ANd VARiance of MRC AND EGC UNDER $\mathbf{H}_{0}$ AND $\mathbf{H}_{1}$ With $K$ SEnSORS

\begin{tabular}{c||c|c}
\hline & MRC & EGC \\
\hline \multirow{2}{*}{$\mathbf{H}_{0}$} & $\mu_{M R C 0}=2 P_{f}-1$ & $\mu_{E G C 0}=\frac{\sqrt{\pi}}{2}\left(2 P_{f}-1\right)$ \\
& $\sigma_{M R C 0}^{2}=\frac{1}{K}\left[1+\sigma^{2}+4 P_{f}\left(1-P_{f}\right)\right]$ & $\sigma_{E G C 0}^{2}=\frac{1}{K}\left[\frac{4-\pi}{4}+\sigma^{2}+\pi P_{f}\left(1-P_{f}\right)\right]$ \\
\hline \multirow{2}{*}{$\mathbf{H}_{1}$} & $\mu_{M R C 1}=2 P_{d}-1$ & $\mu_{E G C 1}=\frac{\sqrt{\pi}}{2}\left(2 P_{d}-1\right)$ \\
& $\sigma_{M R C 1}^{2}=\frac{1}{K}\left[1+\sigma^{2}+4 P_{d}\left(1-P_{d}\right)\right]$ & $\sigma_{E G C 1}^{2}=\frac{1}{K}\left[\frac{4-\pi}{4}+\sigma^{2}+\pi P_{d}\left(1-P_{d}\right)\right]$ \\
\hline
\end{tabular}

8 sensors with sensor level $P_{f}=0.05$ and $P_{d}=0.5$, channel $S N R=5 d B$

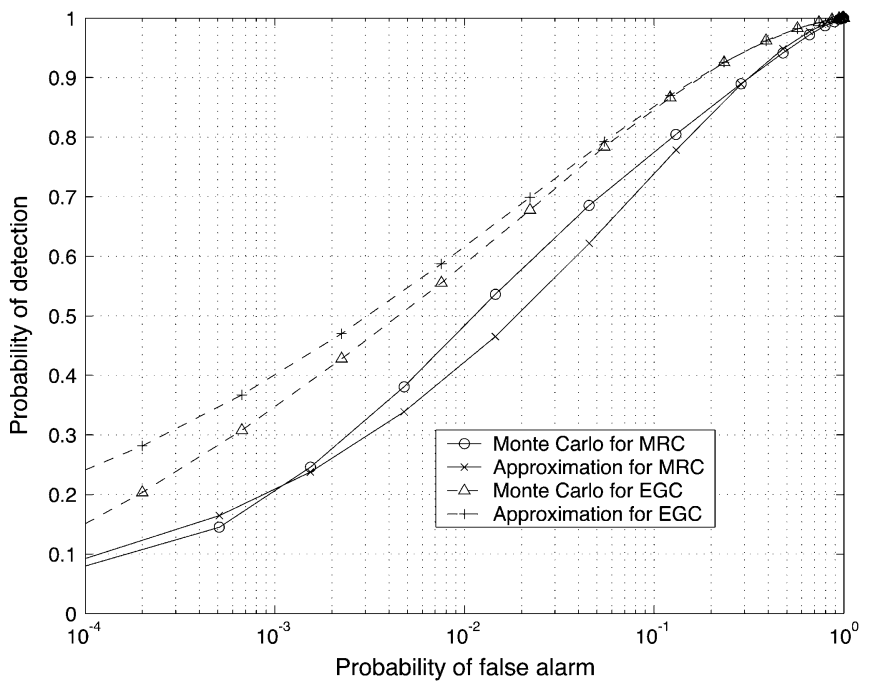

Fig. 2. ROC curves for MRC and EGC obtained by simulation and numerical approximation using the CLT.

using the CLT. In this example, the total number of sensors is 8 with sensor level $P_{f}=0.05$ and $P_{d}=0.5,{ }^{1}$ and the channel SNR is $5 \mathrm{~dB}$. While some discrepancy exists, the approximations using the CLT match relatively well to the simulation results. We have also found through extensive simulations that the accuracy of the CLT approximation not only depends on the number of sensors involved, but also on other system parameters, such as the sensor performance indices $\left(P_{f k}\right.$ and $\left.P_{d k}\right)$ and the channel SNR.

As an alternative to using the ROC curve for performance comparison, one can also resort to the so-called deflection measure [17], which is defined as

$$
D(\Lambda)=\frac{\left[E\left(\Lambda \mid H_{1}\right)-E\left(\Lambda \mid H_{0}\right)\right]^{2}}{\operatorname{Var}\left(\Lambda \mid H_{0}\right)} .
$$

This is in essence the SNR of the detection statistic. This deflection measure can be easily calculated using the first- and second-order moments obtained in Table I. Plotted in Fig. 3 are the deflection values for both MRC and EGC given that the sensor level $P_{f}=0.05$ and $P_{d}=0.5$ with a total number of sensors equal to 8 . Clearly, while MRC has slight advantage over EGC for very low channel SNR, it is dominated by EGC for most channel SNR values.

\section{B. Simulation Results}

In this section, we compare the performance of the four proposed fusion rules using simulations. The sensor level false alarm rate is assumed to be $P_{f}=0.05$, whereas the detection probability is $P_{d}=0.5$.

${ }^{1}$ If the local sensor observes a constant signal in additive white Gaussian noise, then the SNR corresponding to the above $P_{f}$ and $P_{d}$ values is roughly $4.3 \mathrm{~dB}$.

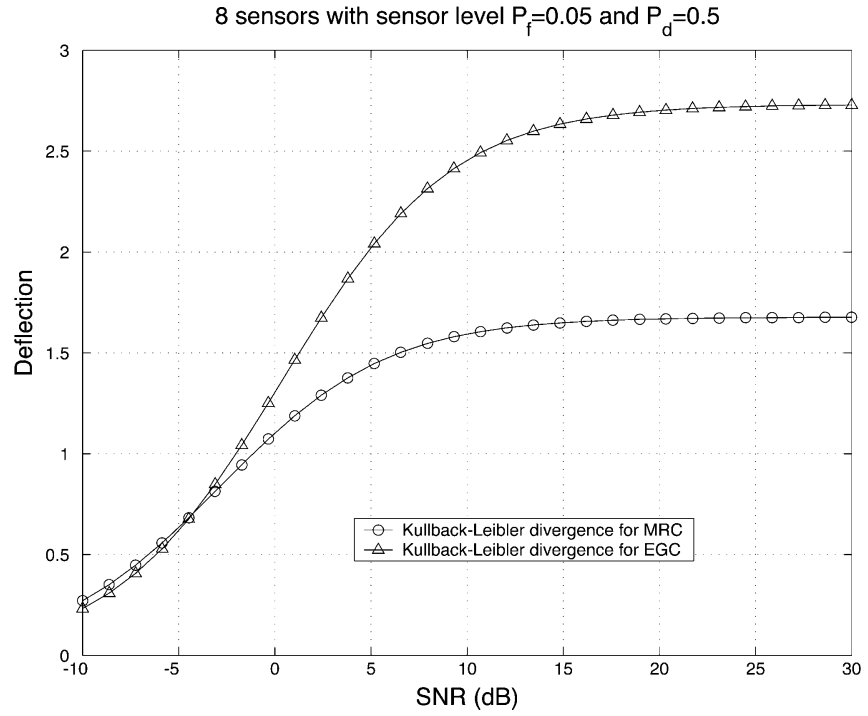

Fig. 3. Deflection measure of MRC and EGC statistics.

System level $P_{f 0}=0.01,8$ sensors with sensor level $P_{f}=0.05$ and $P_{d}=0.5$

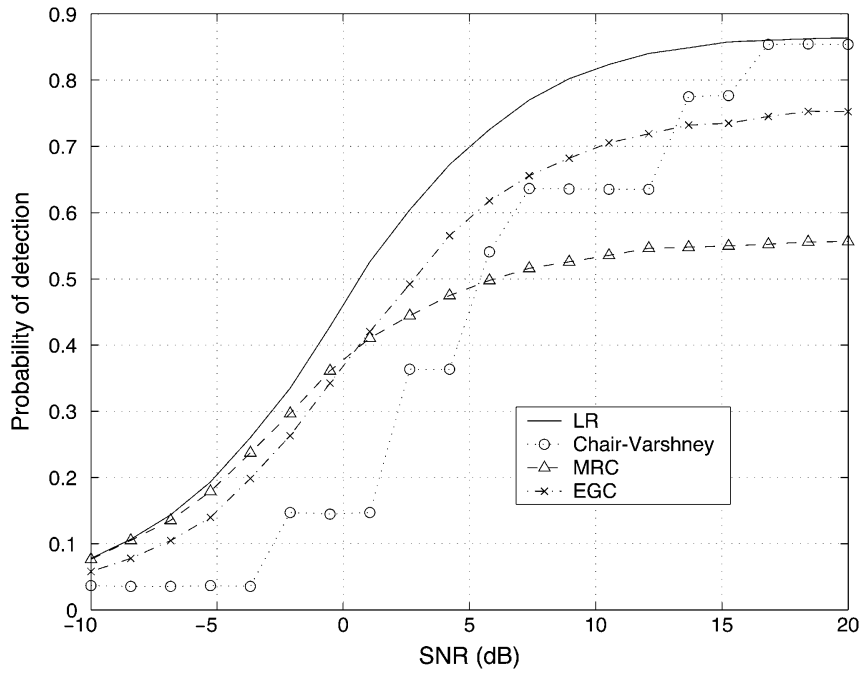

Fig. 4. Probability of detection as a function of channel SNR for Rayleigh fading channels with eight sensors. The system false alarm rate is fixed at $P_{f 0}=$ 0.01 .

The total number of sensors is fixed at 8 . Fig. 4 gives the probability of detection as a function of channel SNR for a fixed system false alarm at $P_{f 0}=0.01$. From this figure, it is easy to see that at very low and high SNR, MRC, and the Chair-Varshney statistic can approach the LR performance quite well, whereas the EGC fusion rule provides the most robust performance among the three suboptimal approaches. 
TABLE II

Performance Comparison Among the Four Fusion Rules For Decisions Transmitted Through Fading Channels

\begin{tabular}{c||c|c}
\hline Fusion rule & a priori information required & Performance \\
\hline \hline$\Lambda$ (LR) & Channel SNR and sensor performance indices & Optimum \\
\hline$\Lambda_{1}$ (Chair-Varshney) & Sensor performance indices & Near-optimal for large SNR \\
\hline$\Lambda_{2}$ (MRC) & Channel SNR & Near-optimal for low SNR \\
\hline$\Lambda_{3}$ (EGC) & None & Robust for most SNR range \\
\hline
\end{tabular}

The tradeoff between detection performance and the requirement on a priori information for each of the fusion schemes is summarized in Table II. Among the three suboptimal alternatives, the EGC statistic provides the most robust detection performance while requiring a minimum amount of information.

\section{CONCLUSION}

Fusion of binary decisions transmitted over fading and noisy channels in the context of WSNs was studied in this correspondence. Based on the canonical parallel fusion structure that incorporates the fading channel, a LR-based fusion rule has been derived. For robust performance in the absence of prior knowledge regarding the local sensors and/or fading channels, several alternatives were proposed. The twostage implementation using the Chair-Varshney fusion rule provides high SNR approximation to the LR-based fusion rule, whereas the statistic in the form of a MRC statistic gives a low SNR approximation. Another heuristic scheme in the form of an EGC statistic was proposed and we demonstrated that it performs better than both the Chair-Varshney approximation and MRC for a wide range of SNR values. Performance evaluation is conducted using both the ROC curve as well as the deflection measure.

Fusion of binary decisions transmitted over fading channels has particularly important applications in low-cost low-power wireless sensor networks. The work reported here was based on the LR fusion with complete channel knowledge. We will investigate, in our future work, decision fusion schemes when only the fading statistics are available. Further, the dual problem to decision fusion, namely the optimal local sensor decision rule in the presence of nonideal transmission channels will also be investigated and reported elsewhere.

\section{REFERENCES}

[1] S. Kumar and D. Shepherd, "SensIT: Sensor information technology for the warfighter," in Proc. Int. Conf. Inform. Fusion, vol. 1, Montreal, QC, Canada, Aug. 2001, pp. TuC1-TuC10.

[2] S. Kumar, F. Zhao, and D. Shepherd, "Special issue on collaborative signal and information processing in microsensor networks," IEEE Signal Processing Mag., vol. 19, pp. 13-14, Mar. 2002.

[3] Z. Chair and P. K. Varshney, "Optimal data fusion in multiple sensor detection systems," IEEE Trans. Aerosp. Electron. Syst., vol. AES-22, pp. 98-101, Jan. 1986.

[4] P. K. Varshney, Distributed Detection and Data Fusion. New York: Springer, 1997.

[5] E. Drakopoulos and C. C. Lee, "Optimum multisensor fusion of correlated local decisions," IEEE Trans. Aerosp. Electron. Syst., vol. 27, pp. 593-605, July 1991.

[6] M. Kam, Q. Zhu, and W. S. Gray, "Optimal data fusion of correlated local decisions in multiple sensor detection systems," IEEE Trans. Aerosp. Electron. Syst., vol. 28, pp. 916-920, July 1992.

[7] B. Chen and P. K. Varshney, "A Bayesian sampling approach for decision fusion using hierarchical models," IEEE Trans. Signal Processing, vol. 50, pp. 1809-1818, Aug. 2002.

[8] F. Gini, F. Lombardini, and L. Verrazzani, "Decentralised detection stratigies under communication constraints," Proc. Inst. Elect. Eng., F: Radar, Sonar, Navigat., vol. 145, pp. 199-208, Aug. 1998.
[9] C. Rago, P. K. Willett, and Y. Bar-Shalom, "Censoring sensors: A low-communication-rate scheme for distributed detection," IEEE Trans. Aerosp. Electron. Syst., vol. 32, pp. 554-568, Apr. 1996.

[10] T. Kasetkasem and P. K. Varshney, "Communication structure planning for multisensor detection systems," Proc. Inst. Elect. Eng., F: Radar, Sonar, Navigat., vol. 148, pp. 2-8, Feb. 2001.

[11] J. Hu and R. Blum, "On the optimality of finite-level quantization for distributed signal detection," IEEE Trans. Inform. Theory, vol. 47, pp. 1665-1671, May 2001.

[12] J. Chamberland and V. V. Veeravalli, "Decentralized detection in sensor networks," IEEE Trans. Signal Processing, vol. 51, pp. 407-416, Feb. 2003.

[13] S. C. A. Thomopoulos and L. Zhang, "Distributed decision fusion with networking delays and channel errors," Inform. Sci., vol. 66, pp. 91-118, Dec. 1992.

[14] T. M. Duman and M. Salehi, "Decentralized detection over multiple-access channels," IEEE Trans. Aerosp. Electron. Syst., vol. 34, pp. 469-476, Apr. 1998.

[15] G. L. Stüber, Principles of Mobile Communication, Second ed. Boston, MA: Kluwer, 2001.

[16] H. L. Van Trees, Detection, Estimation, and Modulation Theory. New York: Wiley, 1968, vol. 1.

[17] B. Picinbono, "On deflection as a performance criterion in detection," IEEE Trans. Aerosp. Electron. Syst., vol. 31, pp. 1072-1081, July 1995. 